\title{
Models of Galaxy Clusters with Thermal Conduction
}

\author{
Nadia L. Zakamska ${ }^{1}$ \\ nadia@astro.princeton.edu \\ Ramesh Narayan ${ }^{1,2}$ \\ rnarayan@cfa.harvard.edu
}

\begin{abstract}
We present a simple model of hot gas in galaxy clusters, assuming hydrostatic equilibrium and energy balance between radiative cooling and thermal conduction. For five clusters, A1795, A1835, A2199, A2390 and RXJ1347.5-1145, the model gives a good description of the observed radial profiles of electron density and temperature, provided we take the thermal conductivity $\kappa$ to be about $30 \%$ of the Spitzer conductivity. Since the required $\kappa$ is consistent with the recent theoretical estimate of Narayan \& Medvedev (2001) for a turbulent magnetized plasma, we consider a conduction-based equilibrium model to be viable for these clusters. We further show that the hot gas is thermally stable because of the presence of conduction. For five other clusters, A2052, A2597, Hydra A, Ser 159-03 and 3C295, the model requires unphysically large values of $\kappa$ to fit the data. These clusters must have some additional source of heat, possibly an active galactic nucleus since all the clusters have strong radio galaxies at their centers. We suggest that thermal conduction, though not dominant in these clusters, may nevertheless play a significant role by preventing the gas from becoming thermally unstable.
\end{abstract}

Subject headings: galaxies: clusters - cooling flows - X-rays: galaxies - conduction

\section{Introduction}

For many years it was thought that radiative losses via X-ray emission in galaxy clusters leads to a substantial inflow of gas, and mass dropout, in the form of a "cooling flow" (see

\footnotetext{
${ }^{1}$ Department of Astrophysical Sciences, Princeton University, Princeton, NJ 08544

${ }^{2}$ Harvard-Smithsonian Center for Astrophysics, 60 Garden Street, Cambridge, MA 02138
} 
Fabian 1994 for a review). Mass deposition rates were estimated to be as much as several hundred $M_{\odot} \mathrm{yr}^{-1}$ in some clusters (e.g., Peres et al. 1998). However, there was little direct evidence for the mass dropout in any band other than X-rays (Fabian 1994).

Recent high resolution X-ray data from XMM-Newton and Chandra indicate that there is no evidence for the multi-temperature gas that one expects if there is substantial mass dropout (Peterson et al. 2001; Tamura et al. 2001; Böhringer et al 2001; Fabian et al. 2001; Molendi \& Pizzolato 2001; Matsushita et al. 2002; Johnstone et al. 2002). The new data strongly suggest that mass dropout must be prevented by some additional source of heat that balances radiative losses. Two possibilities are currently being investigated for the heat source: (i) energy in jets, outflows, and radiation from a central active galactic nucleus (Pedlar et al. 1990; Tabor \& Binney 1993; Churazov et al. 2000, 2002; Ciotti \& Ostriker 2001; Brüggen \& Kaiser 2002), and (ii) thermal energy from outer regions of the cluster transported to the central cooling gas by conduction (Narayan \& Medvedev 2001, hereafter NM01).

Conduction in clusters has been discussed by several authors over the years in various contexts (Binney \& Cowie 1981; Tucker \& Rosner 1983; Bertschinger \& Meiksin 1986; Bregman \& David 1988; Gaetz 1989; Rosner \& Tucker 1989; David et al. 1992; Pistinner \& Shaviv 1996), but its importance was always considered doubtful. For conduction to have any significant effect on the cooling gas in a cluster, the effective isotropic coefficient of conduction $\kappa$ has to be a reasonable fraction of the classical Spitzer (1962) conductivity $\kappa_{S p}$. However, conventional wisdom is that magnetic fields strongly suppress conduction perpendicular to the field. Therefore, while conduction may be very efficient parallel to the field, the overall isotropic $\kappa$ is expected to be $\ll \kappa_{S p}$.

This picture has changed with the recent work of NM01 who, following earlier work by Rechester \& Rosenbluth (1978), Chandran \& Cowley (1998), Chandran et al. (1999) and Malyshkin \& Kulsrud (2001), showed that a turbulent MHD medium in which the fluctuation spectrum extends over two or more decades of spatial scales has an effective $\kappa$ that is a substantial fraction of $\kappa_{S p}$. This is because of chaotic transverse wandering of field lines as a result of strong MHD turbulence (Goldreich \& Sridhar 1995, 1997), which leads to a large enhancement of cross-field diffusion. NM01 estimated that the ratio $f=\kappa / \kappa_{S p}$ in a turbulent MHD medium is approximately 0.2 , though, given the uncertainties in their model, the value could probably lie anywhere in the range $\sim 0.1-0.4$.

NM01 showed via a simple order-of-magnitude estimate that thermal conduction with $f \sim 0.2$ is sufficient to balance the radiative losses of the cooling gas in X-ray clusters. Based on this, they suggested that conduction might be a large part of the explanation for the lack of mass dropout in X-ray clusters. Their suggestion finds support in the work of Voigt et 
al. (2002) and Fabian et al. (2002) who show that conduction with $f$ in the range $0.1-1$ can indeed be a dominant heat source in X-ray clusters except perhaps in the very central regions.

The aim of the present paper is to study conduction in galaxy clusters in more detail. Our goals are two-fold. First, we consider a very simple model in which the gas is in hydrostatic equilibrium and in thermal balance, with cooling exactly compensated by heat conduction. We investigate whether this basic model can fit the observed shapes of the electron density profile $n_{e}(r)$ and temperature profile $T(r)$ of clusters for which high-resolution observations are available. We believe this approach, which is complementary to that of Voigt et al. (2002), is a good test of the conduction hypothesis, and a reliable method of estimating what value of $f$ is needed in various clusters to explain the observations. Second, having found a steady state model for a given cluster, we check whether the hot gas is thermally stable. We briefly discuss the importance and plausibility of other energy sources.

The paper is organized as follows. In $\S 2$ we write down the relevant differential equations and boundary conditions for a steady equilibrium model of hot gas in a cluster. In $\S 3$ we compare model predictions with data on 10 clusters, and in $\S 4$ we discuss the thermal stability of the gas in these clusters. We conclude with a discussion in $\S 5$. Throughout the paper,

we take $H_{0}=70 \mathrm{~km} \mathrm{sec}^{-1} \mathrm{Mpc}^{-1}, \Omega_{M}=0.3$ and $\Omega_{\Lambda}=0.7$, rescaling observational results whenever the original papers have used a different cosmology for their analysis.

\section{The Model}

We consider a spherically symmetric cluster containing hot gas in equilibrium with no inflow. The condition of hydrostatic equilibrium gives

$$
\frac{1}{\rho} \frac{d p}{d r}=-\frac{d \phi}{d r}
$$

where $\rho(r)$ is the gas density, $p(r)$ is the pressure, and $\phi(r)$ is the gravitational potential. We assume that gas pressure dominates and that magnetic fields have a negligible dynamical effect, so that

$$
p=\frac{\rho k_{B} T}{\mu m_{u}}=\frac{\mu_{e}}{\mu} n_{e} k_{B} T \equiv \rho c_{s}^{2},
$$

where $T(r)$ is the temperature of the gas, $n_{e}(r)$ is the electron number density, $\mu$ is the mean molecular weight, $\mu_{e}$ is the molecular weight per electron, $m_{u}$ is the atomic mass unit, and $c_{s}(r)$ is the isothermal sound speed of the gas. We assume that the gas has hydrogen fraction $X=0.7$ and helium fraction $Y=0.28$, so that $\mu=0.62, \mu_{e}=1.18$ and $\mu_{e} / \mu=1.91$ (taking hydrogen and helium to be fully ionized). 
The energy equation of the gas is a simple balance between radiative cooling and heating by thermal conduction. Thus, in steady state, we have

$$
\frac{1}{r^{2}} \frac{d}{d r}\left(r^{2} F\right)=-j,
$$

where $F(r)$ is the heat flux due to electron conduction and $j(r)$ is the rate of radiative cooling per unit volume of the gas. We assume that the conductivity of the gas $\kappa$ is a fixed fraction $f$ of the classical Spitzer (1962) conductivity. Thus,

$$
\begin{gathered}
F=-\kappa \frac{d T}{d r}, \\
\kappa=f \kappa_{S p}=f \frac{1.84 \times 10^{-5} T^{5 / 2}}{\ln \Lambda} \operatorname{erg~s}^{-1} \mathrm{~K}^{-1} \mathrm{~cm}^{-1},
\end{gathered}
$$

where $\ln \Lambda \sim 37$ is the usual Coulomb logarithm. In the relevant range of temperatures the gas cools primarily by thermal bremsstrahlung. We therefore write (Rybicki \& Lightman 1979)

$$
j=2.1 \times 10^{-27} n_{e}^{2} T^{1 / 2} \mathrm{erg} \mathrm{cm}^{-3} \mathrm{~s}^{-1},
$$

where the numerical coefficient corresponds to the particular gas composition that we have assumed; the coefficient also includes an average Gaunt factor.

The gravitational acceleration $d \phi(r) / d r$ due to the mass in the cluster is obtained by solving Poisson's equation,

$$
\frac{1}{r^{2}} \frac{d}{d r}\left(r^{2} \frac{d \phi}{d r}\right)=4 \pi G\left(\rho_{D M}+\rho\right),
$$

where $\rho_{D M}(r)$ is the density distribution of the dark matter, which we assume has the NFW form (Navarro et al. 1997) with a softened core:

$$
\rho_{D M}(r)=\frac{M_{0} / 2 \pi}{\left(r+r_{c}\right)\left(r+r_{s}\right)^{2}} .
$$

Here, $r_{s}$ is the standard scale radius of the NFW profile, $r_{c}$ is a core radius inside which the density becomes constant, and $M_{0}$ is a characteristic mass (e.g., if $r_{c}=0$, the mass inside a sphere of radius $r_{s}$ is $\left.(2 \ln 2-1) M_{0}\right)$.

We relate the parameters of the NFW profile to the temperature $T_{\text {out }}$ of the hydrostatic gas at $r \sim r_{s}$, well outside the cooling region of the cluster. We use the mass-temperature relation from Afshordi \& Cen (2002) and the mass-scale relation from Maoz et al. (1997), so that, once we know $T_{\text {out }}$, we can estimate the virial mass $M_{v i r}$ and $r_{s}$. The former is defined as the mass inside the radius within which the mean density of the dark matter is 
$200 \rho_{\text {crit }}(z)$, where $\rho_{\text {crit }}(z)$ is the critical density of the universe at the redshift of the cluster: $\rho_{\text {crit }}(z)=3 H(z)^{2} / 8 \pi G$. The normalization of the NFW profile is then obtained via a simple calculation:

$$
M_{0}=2 \pi \rho_{\text {crit }}(z) r_{s}^{3}\left(\frac{200}{3}\right) \frac{c^{3}}{\ln (1+c)-c /(1+c)},
$$

where $c$ is the concentration parameter:

$$
c=\frac{1}{r_{s}}\left(\frac{3 M_{v i r}}{4 \pi \cdot 200 \rho_{\text {crit }}(z)}\right)^{1 / 3} .
$$

It is interesting that simulations of cluster formation allow us almost completely to determine the cluster potential from a single observed number, $T_{\text {out }}$. The only remaining uncertainty is the shape of the density distribution in the very inner regions of the cluster, which we parametrize through the core radius $r_{c}$.

The value of the core radius $r_{c}$ is somewhat uncertain. It is zero in the original NFW model, but cluster lensing studies (Tyson et al. 1998; Shapiro \& Iliev 2000) suggest that it may be tens of kpc in some clusters. As explained in $\S 3$ below, we try two values of $r_{c}$ : $r_{c}=0$ and $r_{c}=r_{s} / 20$.

Equations (1), (3), (4) and (7) are four ordinary differential equations for the four variables $n_{e}(r), T(r), F(r)$ and $d \phi(r) / d r$. We solve these equations numerically between $r=0$ and $r=2 r_{s}$ with the following four boundary conditions. Two boundary conditions are taken directly from the observations of a given cluster, namely the temperature $T_{\text {in }}=T(0)$ at the cluster center and $T_{\text {out }}=T\left(2 r_{s}\right)$. For $T_{\text {out }}$ we use the observed temperature at around $r \sim r_{s}$, which is well outside the cooling region in the center. The other two boundary conditions are $F(0)=0$ and $M(0)=0$, where $M(r)$ is the the mass (dark matter plus gas) inside radius $r$.

We note that we make a slight approximation by assuming a perfect steady state. In real clusters with conductive heating, the energy for the cooling ultimately comes from gravity and so the gas in the cluster must slowly settle in the gravitational potential. This effect would be accentuated by conduction to the outside, as discussed by Loeb (2002). We do not include gravitational settling in our model. Instead, we assume that the outer regions of the cluster behave like an infinite isothermal heat source at a temperature of $T_{\text {out }}$. We have experimented with different radii for the outer boundary of our models, from $r=r_{s}$ to $r=5 r_{s}$, and find that the results are not sensitive to this choice. The models discussed below correspond to $r=2 r_{s}$. 


\section{Results}

We have analysed the following 10 clusters for which high angular resolution data are available from Chandra or XMM-Newton or both: A1795, A1835, A2052, A2199, A2390, A2597, Hydra A, RXJ 1347.5-1145, Ser 159-03, and 3C295. Table 1 lists the relevant parameters for each cluster. In the case of several of these clusters, deprojected density and temperature profiles are available, and we have used them in our modeling. In a few cases, however (A2597, A2052, Hydra A, Ser 159-03), the original authors have not published deprojected profiles, and we had to use the un-deprojected data. Given the observational uncertainties, deprojection only affects the profiles in the innermost regions of the cluster, within about $30 \mathrm{kpc}$. On the other hand, our model solutions only depend slightly on the inner temperature. Therefore, we believe that the shapes of the profiles are essentially the same outside about $30 \mathrm{kpc}$, whether we use deprojected or un-deprojected temperatures.

Table 1 lists for each cluster the inner and outer temperatures, $T_{\text {in }}$ and $T_{\text {out }}$, which were used as boundary conditions in the modeling. It also lists the parameters of the NFW profile of the dark matter, which were estimated from $T_{\text {out }}$ as described in $\S 2$. The last column gives references to the original publications for the X-ray data.

We carried out the modeling as follows. We first assumed that the core radius $r_{c}=0$ and selected a value of $f$ (say 0.2 ). We adjusted the inner density $n_{\text {in }}=n_{e}(0)$ of the gas such that on integrating the four differential equations (1), (3), (4) and (7) from $r=0$ to $r=2 r_{s}$, the model temperature agreed with the measured outer temperature $T_{\text {out }}$. We then compared the model profiles of $n_{e}(r)$ and $T(r)$ with the data; we restricted ourselves to a comparison by eye since neither the deprojected data nor the models are reliable enough for a detailed $\chi^{2}$ fit. If the fit was not satisfactory, we tried other values of $f$. If no value of $f$ gave a satisfactory fit, we repeated the process by setting $r_{c}=r_{s} / 20$. (The ratio 20 was selected to be consistent with estimates from lensing; the solutions are insensitive to the exact value.) For most clusters, we obtained a good fit for some choice of $f$ and one of the two values of $r_{c}$ we tried. The values of the best-fit parameters are listed in Table 2.

We consider a model to be acceptable if the model profiles of $n_{e}(r)$ and $T(r)$ agree well with the data and if the required value of $f$ lies within the range $f \sim 0.1-0.4$ predicted by theory (NM01). By this criterion, 5 of the 10 clusters we studied are consistent with a pure thermal conduction model: A1795, A1835, A2199, A2390, and RXJ 1347.5-1145. Figures 1, 2 and 3 show the fits for three of these clusters (A1795, A2199 and A2390).

We consider the good agreement between the model and the data encouraging since the model has very few adjustable parameters. Note in particular that our primary adjustable parameter $f$ affects only the overall normalization of $n_{e}(r)$, but has no effect on the shapes 
of either $n_{e}(r)$ or $T(r)$. The fact that the model curves agree well with the data is thus a significant result. To our knowledge, this kind of modeling of the shapes of profiles has not been reported before. There is a slight inconsistency in the density profile below $10 \mathrm{kpc}$ in A2199 (and also RXJ 1347.5-1145), but this is hardly surprising since the central galaxy is likely to introduce large perturbations to our assumed NFW profile in this region.

Of the 5 clusters for which we obtain acceptable models, we note that A1835 is a complicated case because the Chandra and XMM-Newton data do not agree with each other; indeed, Markevitch (2002) has shown that there are problems with the analysis of both datasets. We obtain a reasonable model for this cluster if we use the density profile from XMM-Newton data (Majerowicz et al. 2002) corrected for flares. For A1795, although both Chandra and XMM-Newton data sets are available (Ettori et al. 2002; Tamura et al. 2001) we do not use the XMM-Newton temperature profile because it is un-deprojected.

The remaining 5 clusters, A2052, A2597, Hydra A, Ser 159-03 and 3C295, all require large values of $f$, generally greater than unity. Since such values are unphysical, we conclude that these clusters must have some other source of energy in addition to thermal conduction. Heating from a central active galactic nucleus is a promising possibility (Churazov et al. 2002; Brüggen \& Kaiser 2002). The idea is supported by the fact that all these clusters possess strong active galactic nuclei (AGN) in their centers which are also all powerful extended radio sources (3C317 in A2052, PKS2322-122 in A2597, 3C218 in Hydra A, PMNJ2313-4243 in Sersic 159-03, and the cluster 3C295 is named after the radio source in its center). Strong extended radio emission is indicative of powerful outflows interacting with the intracluster medium (e.g. Bridle \& Perley 1984). In contrast, of the 5 well-fit clusters, only two (A1795 and A2199) are known to have strong extended radio sources in their centers (4C26.42 and 3C338, respectively).

Mass inflow and dropout is, of course, another possible source of energy, as has been discussed for many years in the context of cooling flows. However, as mentioned in $\S 1$, spectroscopic analyses of Chandra and XMM-Newton data of several clusters have failed to find any evidence for the multi-phase gas expected if there is significant mass inflow and dropout. The observations place an upper limit on the mass accretion rate in a few clusters of about 10-20\% of the nominal value needed to explain the observed X-ray flux (e.g. David et al. 2001; Schmidt et al. 2001; Johnstone et al. 2002). Accretion at this level cannot substantially modify the energy balance, so one needs either conduction or mechanical energy from an AGN to fit the observations. We have computed models with larger levels of mass accretion, at the level of hundreds of $M_{\odot} \mathrm{yr}^{-1}$, and we do find that accretion can then explain the X-ray data. Although the details of accretion and energy release during the mass dropout are uncertain (see different versions of energy balance in Bertschinger \& 
Meiksin 1986 and Sarazin 1988), we find that we can reproduce the observed density and temperature profiles with reasonable assumptions.

\section{Thermal Stability}

To analyze the stability of the steady-state solutions described in $\S 3$, we work with the full time-dependent equations. Equation (1) generalizes to the Euler equation,

$$
\frac{\partial \mathbf{v}}{\partial t}+(\mathbf{v} \cdot \nabla) \mathbf{v}=-\frac{1}{\rho} \nabla p-\nabla \phi,
$$

where $\mathbf{v}$ is the gas velocity. We also have the continuity equation,

$$
\frac{\partial \rho}{\partial t}+\nabla \cdot(\rho \mathbf{v})=0 .
$$

The energy equation (3) now becomes the time-dependent entropy equation,

$$
\rho T\left[\frac{\partial s}{\partial t}+(\mathbf{v} \cdot \nabla) s\right]=-j-\nabla \cdot \mathbf{F}+q^{+},
$$

where the specific entropy $s$ of the gas is defined in terms of the specific internal energy $\epsilon$ and specific enthalpy $w$ by

$$
T d s=d \epsilon-\frac{p}{\rho^{2}} d \rho=d w-\frac{d p}{\rho} .
$$

For an ideal gas,

$$
\epsilon=\frac{1}{(\gamma-1)} c_{s}^{2}, \quad w=\frac{\gamma}{(\gamma-1)} c_{s}^{2},
$$

where $\gamma$ is the adiabatic index and $c_{s}$ is the isothermal sound speed (Eq. 2). In our calculations, we set $\gamma=5 / 3$.

For completeness, we have included in equation (13) a heating term $q^{+}$in addition to the term due to thermal conduction. This term may refer, for instance, to heating from a central AGN. We assume that the heating rate per unit volume has a power-law dependence on the density,

$$
q^{+}=q_{0}^{+} \rho^{\alpha} .
$$

The heat flux equation (4) and the Poisson equation (7) are still valid, except that we generalize them to include non-spherical perturbations:

$$
\mathbf{F}=-\kappa \nabla T,
$$




$$
\nabla^{2} \phi=4 \pi G\left(\rho_{D M}+\rho\right) .
$$

The above five equations, (11), (12), (13), (17) and (18), describe the general timedependent evolution of the gas. Note that, while we include the effect of a turbulent magnetic field on conduction through the parameter $f<1$ in equation (5), we do not include magnetic pressure or tension in the Euler equation (see Loewenstein 1990 for a discussion of these effects).

For the stability analysis, we take a steady state spherical solution of the equations, e.g., one of the cluster models described in $\S 3$, and consider local linear perturbations of the solution within the WKB approximation; that is, we assume that the perturbations are small and are proportional to $\exp (i k x-i \omega t)$. Balbus (1988) has shown that radial perturbations, with wavevector $k$ parallel to the local direction of gravity, are not well-described by a local analysis; these perturbations also do not show thermal instability. In the following we therefore consider only tangential perturbations, with $k$ and the corresponding $x$ taken to be in a tangential direction with respect to the radius vector.

We carry out the linear stability analysis in the standard way; see Field (1965) for a classic treatment of the thermal stability problem. In brief, we linearize the equations, substitute perturbations of the form $\exp (i k x-i \omega t)$ and derive the dispersion relation for the mode frequency $\omega$. If any of the solutions for $\omega$ has a positive imaginary part, the corresponding mode grows with time and the system is unstable. If all modes for all allowed values of $k$ have $\operatorname{Im}(\omega) \leq 0$, we say that the system is stable.

Before presenting the results, we note that there are several physical effects present in this problem, each with a characteristic frequency or time scale. In addition to the acoustic frequency $c_{s} k$, we have frequencies associated with gravity (Jeans frequency $\omega_{J}$ ), cooling $\left(\omega_{\text {cool }}\right)$ and heating $\left(\omega_{\text {heat }}\right)$, and a characteristic time scale associated with conduction $\left(\tau_{\text {cond }}\right)$ :

$$
\omega_{J}=(4 \pi G \rho)^{1 / 2}, \quad \omega_{\text {cool }}=\frac{j}{\rho c_{s}^{2}}, \quad \omega_{\text {heat }}=\frac{q^{+}}{\rho c_{s}^{2}}, \quad \tau_{\text {cond }}=\frac{\kappa T}{\rho c_{s}^{4}} .
$$

Each of the parameters, $\omega_{J}, \omega_{\text {cool }}, \omega_{\text {heat }}$ and $\tau_{\text {cond }}$, has been defined in such a way that the parameter goes to 0 as the corresponding physical effect becomes negligible.

We discuss first a simple case in which we assume that conduction is negligible ( $\tau_{\text {cond }} \rightarrow$ $0)$ and, for simplicity, we ignore self-gravity $\left(\omega_{J} \rightarrow 0\right)$. Energy balance of the equilibrium system requires $q^{+}=j$, and thus $\omega_{\text {heat }}=\omega_{\text {cool }}$. Assuming that $q^{+}$and $j$ scale with density and temperature as in equations (16) and (6), and taking $\gamma=5 / 3$, we then obtain the following dispersion relation for $\omega$ :

$$
(-i \omega)^{3}+\frac{1}{3} \omega_{\text {cool }}(-i \omega)^{2}+\frac{5}{3} c_{s}^{2} k^{2}(-i \omega)=\left(1-\frac{2}{3} \alpha\right) \omega_{\text {cool }} c_{s}^{2} k^{2} .
$$


By inspection we see that one of the solutions for $\omega$ is always positive imaginary if $\alpha<1.5$. Thus, if $\alpha<1.5$, the system is always thermally unstable. Physically, we expect many heating mechanisms to be described by $\alpha$ in the range between 0 (constant heating rate per unit volume) and 1 (constant heating rate per unit mass). According to equation (20), such systems will be thermally unstable. This suggests that models which try to solve the cooling flow problem by invoking local heating (Churazov et al. 2002; Brüggen \& Kaiser 2002) may still have some difficulties. While they allow equilibrium configurations, the equilibria they obtain are likely to be unstable. Unless the heating occurs preferentially in high density regions, with an index $\alpha \geq 1.5$ (which seems implausible), these models require thermal conduction or some other agency for stability.

We move next to the problem of interest to this paper, namely models in which cooling is balanced entirely by heat conduction $\left(q^{+}=0, \omega_{\text {heat }}=0\right)$. The dispersion relation now takes the form (again setting $\gamma=5 / 3$ )

$$
\begin{gathered}
\frac{3}{2} \omega^{3}+\omega^{2}\left(\frac{1}{2} i \omega_{\text {cool }}+i k^{2} c_{s}^{2} \tau_{\text {cond }}\right)+\omega\left(-\frac{5}{2} k^{2} c_{s}^{2}+\frac{3}{2} \omega_{J}^{2}\right)+ \\
+\frac{3}{2} i \omega_{\text {cool }} k^{2} c_{s}^{2}+\frac{1}{2} i \omega_{\text {cool }} \omega_{J}^{2}-i k^{4} c_{s}^{4} \tau_{\text {cond }}+i k^{2} c_{s}^{2} \tau_{\text {cond }} \omega_{J}^{2}=0
\end{gathered}
$$

Analysis of this dispersion relation shows that an instability is present only when the condition

$$
k^{2} c_{s}^{2}<\frac{1}{2}\left(\omega_{J}^{2}+\frac{3}{2} \frac{\omega_{\text {cool }}}{\tau_{\text {cond }}}+\sqrt{\omega_{J}^{4}+5 \frac{\omega_{J}^{2} \omega_{\text {cool }}}{\tau_{\text {cond }}}+\frac{9}{4} \frac{\omega_{\text {cool }}^{2}}{\tau_{\text {cond }}^{2}}}\right)
$$

is satisfied.

We see that, as expected (e.g., Medvedev \& Narayan 2002), conduction eliminates the thermal instability for short wavelength modes. Physically, the reason is that the shorter the wavelength the larger the temperature gradient for a given mode amplitude; since conduction tries to iron out temperature fluctuations and the conductive flux is proportional to the temperature gradient, short wavelength modes are stabilized. For long wavelengths, however, sound waves can no longer stabilize the gravitational Jeans instability, and (finite) conduction cannot stabilize the thermal instability. This is the physical meaning of the upper limit on $k$ given in equation $(22)$.

Let us denote the critical wavevector for instability by $k_{\text {crit }}$ and the corresponding wavelength by $\lambda_{\text {crit }}=2 \pi / k_{\text {crit }}$. The relevant question is the following: can an unstable mode with wavelength $\lambda_{\text {crit }}$ or larger fit within the system? Recall that our modes are assumed to be in the tangential direction and we carried out the analysis under a local WKB approximation. For this analysis to be meaningful, the wavelength of the mode must be substantially smaller than the circumference $2 \pi r$. A given cluster model is thus stable if $\lambda_{\text {crit }}$ is greater than the largest wavelength for which the WKB analysis is valid, which is probably around $\pi r$. 
Figure 4 shows the variation of $\lambda_{\text {crit }} / \pi r$ with $r$ for the 5 clusters for which we obtained satisfactory models with conduction $(\S 3)$. We see that all 5 clusters are thermally stable according to the criterion described above. One interesting feature is that all the clusters show very similar minimal values of $\lambda_{\text {crit }} / \pi r$ which appear to be fairly close to the threshold of instability. Such a situation would be natural if the clusters, to begin with, had more hot gas than they have now and were thermally unstable. One expects gas to have cooled and dropped out early in the life of the cluster so that the system naturally reached, and has since maintained, a marginally stable configuration. A global stability analysis would need to be carried out to check this idea in more detail.

\section{Discussion}

NM01 found that thermal conduction with $f \equiv \kappa / \kappa_{S p}$ of order a few tenths can explain the gross energetics of hot gas in galaxy clusters. They showed that the heat flux transported to the cooler central regions of the cluster from further out is roughly consistent with what is needed to replace the energy lost through radiative cooling. Gruzinov (2002) and Fabian et al. (2002) confirmed this conclusion.

In this paper, we have carried out a further test of the conduction model by investigating the shapes of the density and temperature profiles, $n_{e}(r)$ and $T(r)$, in an equilibrium cluster. We solve for these profiles self-consistently, as compared to previous authors, e.g., Bertschinger \& Meiksin (1986); Bregman \& David (1988); Voigt et al. (2002) and Fabian et al. (2002), who either used the observations directly or employed simple analytical expressions for the shapes of $n_{e}(r)$ and/or $T(r)$.

Among the 10 clusters that we have studied, 5 clusters, namely, A1795, A1835, A2199, A2390 and RXJ1347.5-1145, can be fitted well with a pure conduction model and with values of $f \sim 0.2-0.4$ (Table 2, Figs. 1, 2, 3). Since the model involves no fitting parameters other than $f$, which is used primarily to set the normalization of $n_{e}$, and $r_{c}$, which is not really a fitting parameter but rather is given one of two values ( $\S 3$ ), the good agreement between the model profiles of $n_{e}(r)$ and $T(r)$ and the data is very encouraging. For these five clusters, the best-fit values of $f$ lie within the range expected on theoretical grounds (NM01). We believe that these are strong arguments in support of the conduction model. We should note, however, that the theoretical estimate $f \sim 0.1-0.4$ obtained by NM01 is based on a specific (plausible) model of the magnetic field topology, suggested by the work

of Goldreich \& Sridhar $(1995,1997)$, but the answer might change substantially if the field topology (which is poorly understood in clusters) is very different. 
The other 5 clusters, namely A2052, A2597, Hydra A, Ser 159-03 and 3C295, require larger values of $f$, of order unity or greater, and we consider them to be inconsistent with a pure conduction model. It is interesting that these five clusters all have active nuclei with strong radio activity, so that nuclear activity might well supply the additional heat required by our models. It is unclear exactly how an AGN would heat the cluster medium. Turbulent mixing of hot gas in a jet with the surrounding cooler gas is a possibility (Churazov et al. 2002; Brüggen \& Kaiser 2002), though the effect of magnetic fields on this mixing is poorly understood. Heating of the gas by cosmic rays (Loewenstein et al. 1991) or hard X-rays (Ciotti \& Ostriker 2001) from the AGN are other possibilities.

In $\S 4$ we showed by means of a local linear stability analysis that the five clusters for which we have obtained good fits with a pure conduction model are all thermally stable. This is an interesting result because, without conduction, cooling gas in clusters is generally thermally unstable. We find it reassuring that a single mechanism, namely conduction, is able both to supply the heat lost via radiative cooling and to control the thermal instability in these clusters. In the other five clusters, which require an additional source of energy such as a central AGN, the thermal stability of the gas is not assured. One way of ensuring stability is to have a heat source with a strong non-linear dependence on density, e.g., $\alpha>1.5$ in equation (16); this appears somewhat unnatural. Alternatively, conduction, though not an important energy source in these clusters, might still play a role in controlling the instability.

Based on the above results, we suggest that AGN heating and conduction both are important in clusters. In some clusters, AGN heating dominates and conduction plays a secondary, though still important, role in helping to stabilize the system. In other clusters, AGN activity is weak, and conduction supplies the energy for cooling as well as prevents the gas from becoming unstable.

While our work suggests that conduction is effective over much of the volume of a cluster, we note that Chandra observations have revealed sharp temperature jumps in some clusters (e.g., Markevitch et al. 2000; Vikhlinin, Markevitch \& Murray 2001ab). These cold fronts are associated with the interface between the intracluster medium and an infalling galaxy or group. The observations clearly indicate that conduction is strongly suppressed across the fronts. Vikhlinin et al. (2001b) have argued that the magnetic field is stretched out parallel to the cold front by the motion of the infalling galaxy/group and that this explains the very low conductivity. Mazzotta et al. (2002) present interesting evidence for a possible KelvinHelmholtz instability in the cold front in A3667, confirming that the fronts are probably transient features with lifetimes of only a couple of dynamical times. The presence of these fronts is thus unlikely to affect the strong conduction that we have hypothesized over the bulk of the cluster. If cold fronts survive for longer than the cooling time of the gas, then the 
thermally isolated cool gas would experience runaway cooling. Since such runaway cooling is ruled out by the absence of substantial mass dropout, the lifetimes of cold fronts cannot be longer than the cooling time.

The authors thank Bill Forman, Christine Jones Forman and Larry David for useful discussions, and the referee for helpful comments on the manuscript. RN thanks the National Science Foundation for support under grant AST 9820686.

\section{REFERENCES}

Afshordi, N., \& Cen, R. 2002 ApJ, 564, 669

Allen, S.W., Taylor, G.B., Nulsen, P.E.J., et al. 2001(a), MNRAS, 324, 842

Allen, S. W., Ettori, S., \& Fabian, A. C. 2001(b) MNRAS, 324, 877

Allen, S.W., Schmidt, R.W., \& Fabian, A.C. 2002, MNRAS, in press (astro-ph/0111368)

Balbus, S.A. 1988, ApJ, 328, 395

Bertschinger, E., \& Meiksin, A. 1986, ApJ, 306, L1

Binney, J., \& Cowie, L.L. 1981, ApJ, 247, 464

Blanton, E.L., Sarazin, C.L., McNamara, B.R., \& Wise, M.W. 2001, ApJ, 558, L15

Böhringer, H. et al. 2001, A\&A, 365, L181

Bregman, J.N., \& David, L.P. 1988, ApJ, 326, 639

Bridle, A.H., \& Perley, R.A. 1984, ARA\&A, 22, 319

Brüggen, M., \& Kaiser, C.R. 2002, Nature, 418, 301

Chandran, B.D.G., \& Cowley, S.C. 1998, Phys. Rev. Lett., 80, 3077

Chandran, B.D.G., Cowley, S.C., Ivanushkina, M., \& Sydora, R. 1999, ApJ, 525, 638

Churazov, E., Forman, W., Jones, C., \& Bohringer, H. 2000, A\&A, 356, 788

Churazov, E., Sunyaev, R., Forman, W., \& Böhringer, H. 2002, MNRAS, 332, 729

Ciotti, L., \& Ostriker, J.P. 2001, ApJ, 551131 
David, L.P., Hughes, J.P., \& Tucker, W.H. 1992, ApJ, 394, 452

David, L.P., Nulsen, P.E.J., McNamara, B.R., Forman, W., Jones, C., Ponman,T., Robertson, B., \& Wise, M. 2001, ApJ, 557, 546

Gruzinov, A. 2002, astro-ph/0203031

Ettori, S., Fabian, A.C., Allen, S.W., \& Johnstone, R.M. 2002, MNRAS, in press (astroph/0111586)

Fabian, A.C. 1994, ARA\&A, 32, 277

Fabian, A.C., Mushotzky, R.F., Nulsen, P.E.J., \& Peterson, J.R. 2001, MNRAS, 321, L20

Fabian, A.C., Voigt, L.M., \& Morris, R.G., 2002, MNRAS, submitted (astro-ph/0206437)

Field, G.B. 1965, ApJ, 142, 531

Gaetz, T.J. 1989, ApJ, 345, 666

Goldreich, P., \& Sridhar, S. 1995, ApJ, 438, 763

Goldreich, P., \& Sridhar, S. 1997, ApJ, 485, 680

Johnstone, R.M., Allen, S.W., Fabian, A.C., \& Sanders, J.S. 2002, MNRAS, in press (astro$\mathrm{ph} / 0202071)$

Kaastra, J.S., Ferringno, C., Tamura, T., Paerels, F.B.S., Peterson, J.R., \& Mittaz, J.P.D. 2001, A\&A, 365, L99

Loeb, A. 2002, New Astr., 7, 279

Loewenstein, M. 1990, ApJ, 349, 471

Loewenstein, M., Zweibel, E.G., \& Begelman, M.C. 1991, ApJ, 377, 392

Majerowicz, S., Neumann, D.M., \& Reiprich, T.H. 2002, A\&A, submitted (astro$\mathrm{ph} / 0202347)$

Malyshkin, L., \& Kulsrud, R. 2001, ApJ, 549, 402

Maoz, D., Rix, H.-W., Gal-Yam, A., \& Gould, A. 1997, ApJ, 486, 75

Markevitch, M. et al. 2000, ApJ, 541, 542

Markevitch, M. 2002 (astro-ph/0205333) 
Matsushita, K. Belsole, E., Finoguenov, A., Bohringer, H. 2002, A\&A, 386, 77

Mazzotta, P., Vikhlinin, A., Fusco-Femiano, R., \& Markevitch, M. 2002, ApJ, 569, L31

McNamara, B.R., Wise, M.W., Nulsen, P.E.J. et al 2001, ApJ, 562, L149

Medvedev, M.V., \& Narayan, R. 2002, MNRAS, submitted (astro-ph/0107066)

Molendi, S., \& Pizzolato, F. 2001, ApJ, 560, 194

Narayan, R., \& Medvedev, M.V. 2001, ApJ, 562, L129 (NM01)

Navarro, J.F., Frenk, C.S., \& White, S.D.M. 1997, ApJ, 490, 493

Pedlar, A., Ghataure, H.S., Davies, R.D., Harrison, B.A., Perley, R., Crane, P.C., \& Unger, S.W. 1990, MNRAS, 246, 477

Peres, C.B., Fabian, A.C., Edge, A.C., Allen, S.W., Johnstone, R.M., \& White, D.A. 1998, MNRAS, 298, 416

Peterson, J.R., Paerels, F.B.S., Kaastra, J.S., et al. 2001, A\&A, 365, L104

Pistinner, S., and Shaviv, G. 1996, ApJ, 459, 1

Rechester, A.B., \& Rosenbluth, M.N. 1978, Phys. Rev. Lett., 40, 38

Rosner, R., \& Tucker, W.H. 1989, ApJ, 338, 761

Rybicki, G.B., \& Lightman, A.P. 1979, Radiative processes in astrophysics (New York: Wiley)

Sarazin, C.L. 1988, X-ray emissions from clusters of galaxies (Cambridge: Cambridge University Press)

Schmidt, R.W., Allen, S.W., \& Fabian, A.C. 2001, MNRAS, 327, 1057

Shapiro, P.R., \& Iliev, I.T. 2000, ApJ, 542, L1

Spitzer, L. 1962, Physics of Fully Ionized Gases (New York: Interscience)

Tabor, G., \& Binney, J. 1993, MNRAS, 263, 323

Tamura, T., Kaastra, J.S., Peterson, J.R., Paerels, F., et al. 2001, A\&A, 365, L87

Tucker, W.H., \& Rosner, R. 1983, ApJ, 267, 547 
Tyson, J.A., Kochanski, G.P., \& Dell'Antonio, I.P. 1998, ApJ, 498, L107

Vikhlinin, A., Markevitch, M., \& Murray, S. S. 2001a, ApJ, 549, L47

Vikhlinin, A., Markevitch, M., \& Murray, S. S. 2001b, ApJ, 551, 160

Voigt, L.M., Schmidt, R.W., Fabian, A.C., Allen, S.W., \& Johnstone R.M., 2002, MNRAS, submitted (astro-ph/0203312) 

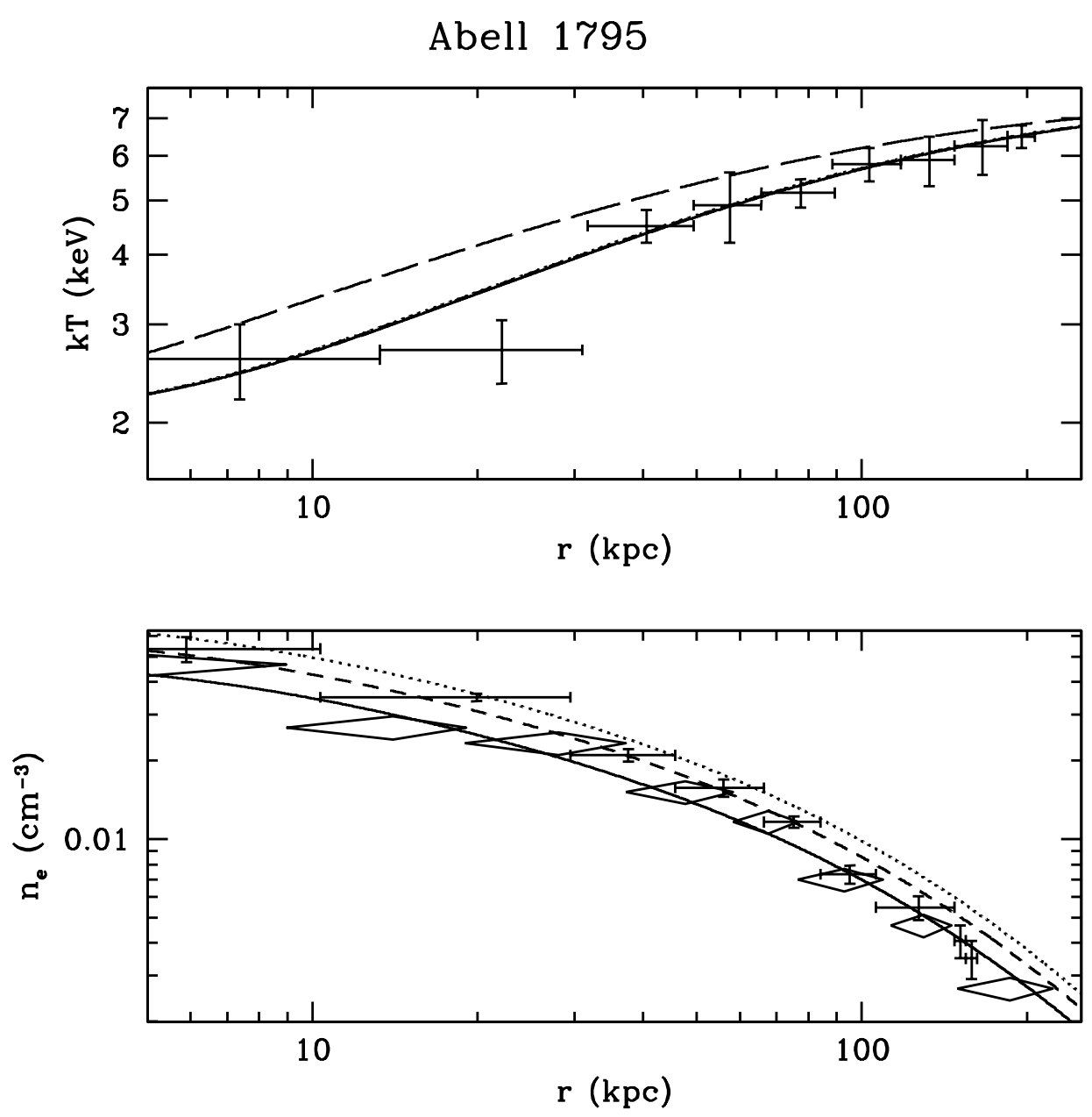

Fig. 1.- Observed and modeled temperature and density profiles for A1795. Crosses correspond to Chandra data (Ettori et al. 2002) and diamonds to XMM - Newton data (Tamura et al. 2001). The solid, dashed and dotted lines refer to models with $r_{c}=r_{s} / 20$ and different conductivity coefficients, $f=0.2,0.3,0.4$, respectively. Note that changing the conductivity has no effect on the temperature profile. In the case of the density profile, $f$ changes the normalization but not the shape. The long-dashed line shows the temperature profile for a model in which $r_{c}=0$ and $f=0.4$. 

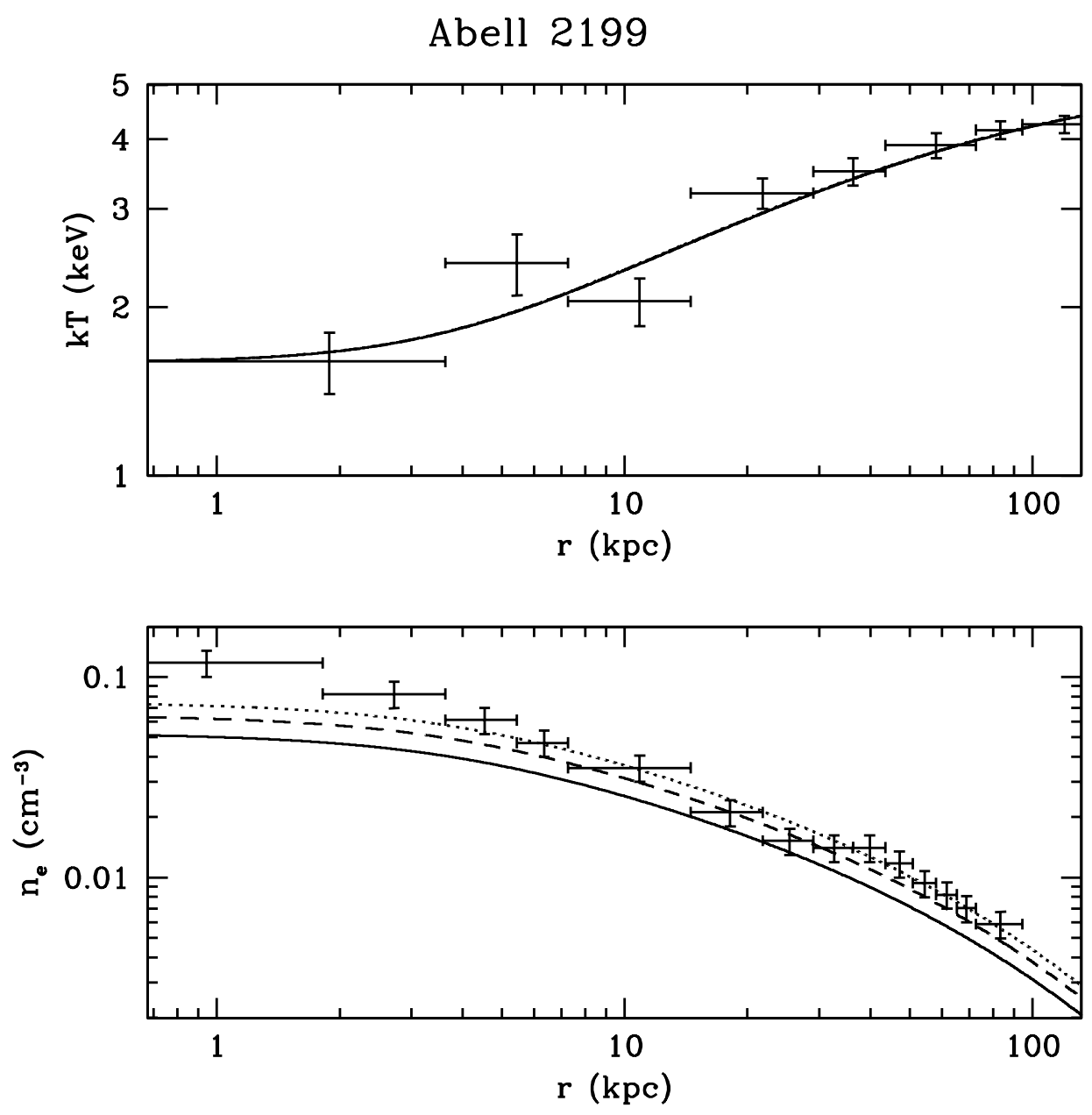

Fig. 2.- Observed and modeled temperature and density profiles for A2199. Crosses indicate Chandra data (Johnstone et al. 2002). The solid, dashed and dotted lines refer to models with $r_{c}=0$ and $f=0.2,0.3,0.4$, respectively. 

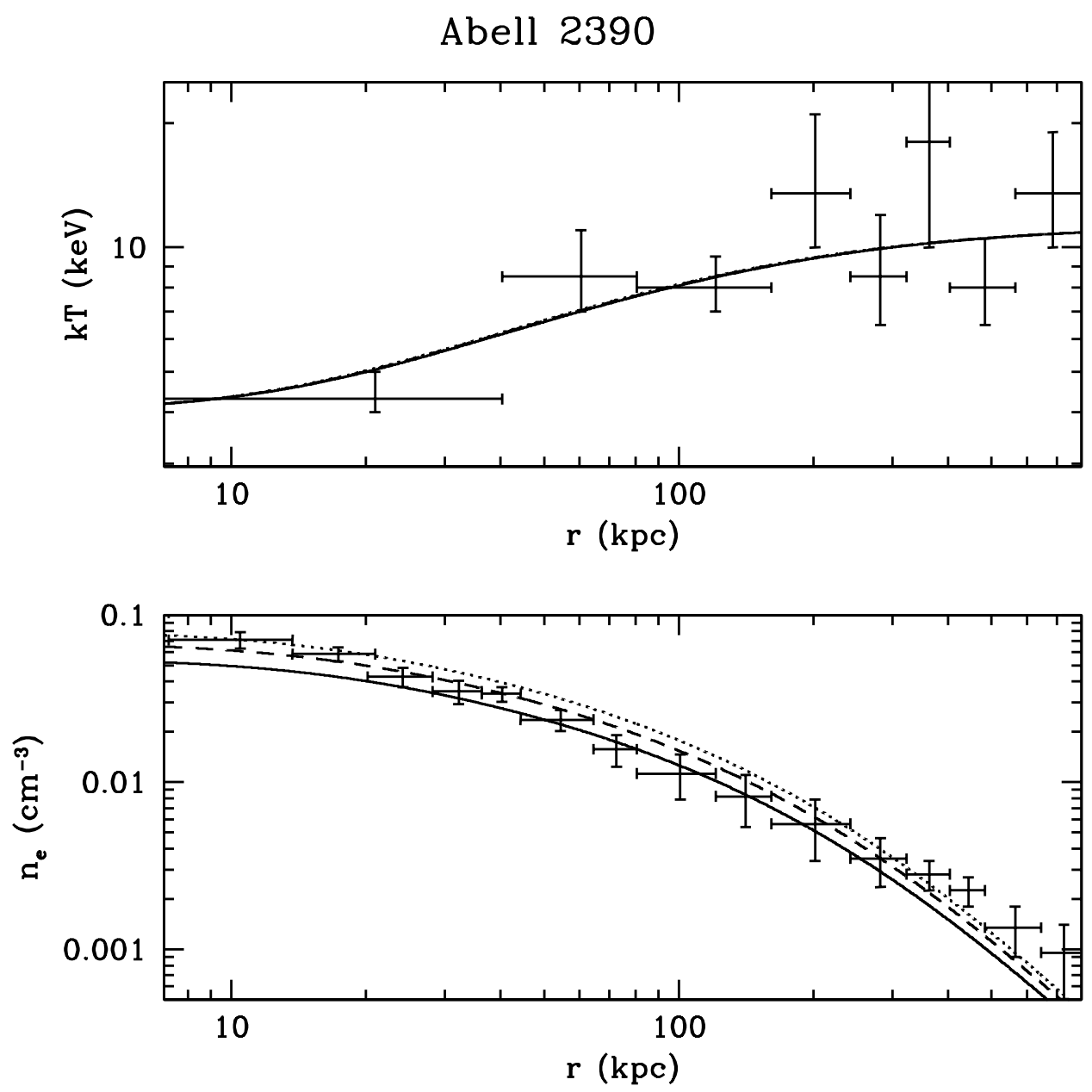

Fig. 3.- Observed and modeled temperature and density profiles for A2390. Crosses indicate Chandra data (Allen et al. 2001b). The solid, dashed and dotted lines refer to models with $r_{c}=r_{s} / 20$ and $f=0.2,0.3,0.4$, respectively. 


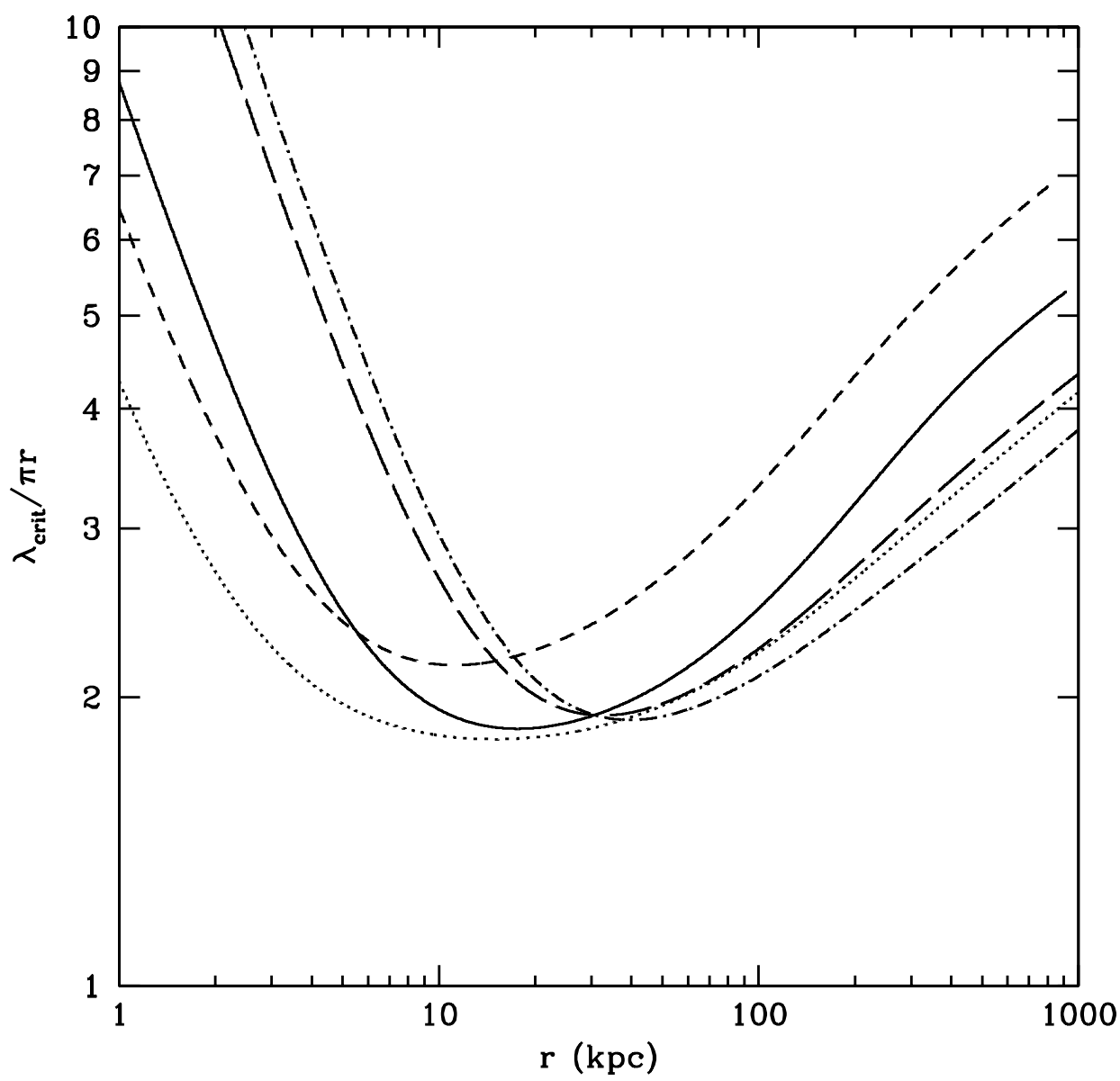

Fig. 4.- Results of a local stability analysis. The stability parameter $\lambda_{\text {crit }} / \pi r$ is shown as a function of radius for 5 clusters that are fit well by a conduction model. The solid line corresponds to A1795, the dotted line to A1835, the short-dashed line to A2199, the long-dashed line to A2390, and the dotted-dashed line to RXJ1347.5-1145. Note that all the curves have similar shapes, with minima around 10-40 kpc. A given system will be unstable if $\lambda / \pi r$ is less than or of order unity at any radius. By this criterion, all five systems are stable. 
Table 1.

Observational and cosmological parameters of the clusters.

\begin{tabular}{ccccccc}
\hline Name & $T_{\text {in }}(\mathrm{keV})$ & $T_{\text {out }}(\mathrm{keV})$ & $M_{\text {vir }}\left(M_{\odot}\right)$ & $r_{s}(\mathrm{kpc})$ & $M_{0}\left(M_{\odot}\right)$ & ref \\
\hline \hline Abell 1795 & 2 & 7.5 & $1.2 \times 10^{15}$ & 460 & $6.6 \times 10^{14}$ & $(1,2)$ \\
Abell 1835 & 5 & 11 & $1.7 \times 10^{15}$ & 520 & $1.1 \times 10^{15}$ & $(3)$ \\
& 4 & 8 & $1.1 \times 10^{15}$ & 470 & $8.8 \times 10^{14}$ & $(4,5)$ \\
Abell 2052 & 1.3 & 3.5 & $3.9 \times 10^{14}$ & 340 & $2.3 \times 10^{14}$ & $(6)$ \\
Abell 2199 & 1.6 & 5 & $6.6 \times 10^{14}$ & 390 & $3.8 \times 10^{14}$ & $(7)$ \\
Abell 2390 & 4 & 11 & $1.7 \times 10^{15}$ & 520 & $1.1 \times 10^{15}$ & $(8)$ \\
Abell 2597 & 1 & 4 & $4.6 \times 10^{14}$ & 360 & $2.8 \times 10^{14}$ & $(9)$ \\
Hydra A & 3 & 4.1 & $4.9 \times 10^{14}$ & 370 & $2.9 \times 10^{14}$ & $(10)$ \\
RXJ 1347.5-1145 & 6 & 16 & $2.5 \times 10^{15}$ & 600 & $1.8 \times 10^{15}$ & $(11)$ \\
Sersic 159-03 & 2 & 2.7 & $2.6 \times 10^{14}$ & 310 & $1.6 \times 10^{14}$ & $(12)$ \\
3C295 & 2 & 6 & $5.8 \times 10^{14}$ & 420 & $4.9 \times 10^{14}$ & $(13)$ \\
\hline
\end{tabular}

References: (1) Ettori et al. (2002); (2) Tamura et al. (2001); (3) Schmidt et al. (2001); (4) Markevitch (2002); (5) Majerowicz et al. (2002); (6) Blanton et al. (2001); (7) Johnstone et al. (2002); (8) Allen et al. (2001b); (9) McNamara et al (2001); (10) David et al. (2001);

(11) Allen et al. (2002); (12) Kaastra et al. (2001); (13) Allen et al. (2001a).

Notes: Observed parameters ( $T_{i n}$ is the temperature in the innermost region of the cluster, $T_{\text {out }}$ is the temperature outside the cooling region) and inferred parameters of the NFW profile (the virial mass of the cluster, $M_{v i r}$; the scale radius $r_{s}$ and the characteristic mass $\left.M_{0}\right)$; see $\S 2$ on how the NFW parameters were obtained. The last column contains references to the original publications for the X-ray data on the gas temperature and electron density profiles. 
Table 2.

Best-fit parameters of the conduction model.

\begin{tabular}{ccccl}
\hline Name & $\mathrm{f}$ (best-fit) & $n_{e}(0)\left(\mathrm{cm}^{-3}\right)$ & $r_{c}(\mathrm{kpc})$ & \\
\hline \hline Abell 1795 & 0.2 & 0.049 & $r_{s} / 20$ & (see Fig. 1) \\
Abell 1835 & 0.4 & 0.17 & $r_{s} / 20$ & \\
Abell 2052 & 0.6 & 0.089 & 0 & \\
Abell 2199 & 0.4 & 0.074 & 0 & (see Fig. 2) \\
Abell 2390 & 0.3 & 0.069 & $r_{s} / 20$ & (see Fig. 3) \\
Abell 2597 & 2.4 & 0.13 & $r_{s} / 20$ & \\
Hydra A & 1.5 & 0.020 & 0 & \\
RXJ 1347.5-1145 & 0.3 & 0.11 & $r_{s} / 20$ & \\
Sersic 159 & 5.6 & 0.042 & 0 & \\
3C295 & 1 & 0.084 & $r_{s} / 20$ & \\
\hline
\end{tabular}

Notes: $f=\kappa / \kappa_{S p}$ is the value of the conductivity coefficient required to reproduce the observed electron density, $n_{e}(0)$ is the corresponding model electron density in the center, and $r_{c}$ is the core radius adjusted to fit the observed temperature profile, for which we only consider two values (see $\S 3$ ). 